Received: April 06, 2017

Accepted: April 15, 2017

Published: April 25, 2017

\title{
Non Typical Risk Factors in Atherosclerosis and Coro- nary Disease. It Is Time to Open the Box and Look Out- side
}

\author{
Sergio Mejia Viana* \\ Medical Investigation Unit, St Bernard's Hospital, Harbour Views Road, GX11 1AA, \\ Gibraltar.
}

*Corresponding author: Sergio Mejia Viana, Medical Investigation Unit, St Bernard's Hospital, Harbour Views Road, GX11 1AA, Gibraltar, E-mail: sergio.mejia@gha.gi

\section{Abstract}

Since Framingham we are assuming that age, smoking, hypertension, diabetes, obesity and family history are the main risk factors for cardiovascular disease, stroke and atherosclerosis. In spite of aggressive strategies, medication for cholesterol, diabetes and hypertension, and low fat diet approach, the incidence of cardiovascular disease is on the rise in western countries. Moreover, life expectancy in the USA is for the first time falling down in the last decades. Alternative approaches has claim the importance of heavy metals chronic exposure, lack of Vitamin C and Vitamin K, periodontal disease and stress as fundamental risk factors and coadjutants for atherosclerosis. Some of these so called atypical factors are described in this article.

\section{Keywords}

Atherosclerosis; Risk factors; Low ascorbate; Vitamin K2, Periodontal disease; Prevention;

\section{Atherosclerosis and Low Ascorbate Levels}

Atherogenesis is a multifactorial pathophysiological process of the arterial vasculature, which is characterized by progression from inflammation and smooth muscle cell proliferation to late stages that are marked by thrombotic and fibrotic obliterations of the vessels. Examination of the earliest cellular events than occur during atherogenesis has demonstrated a specialized type of chronic inflammatory response that precedes migration and proliferation of arterial smooth muscle cells. The first observable events include increased accumulation of lipid and lipoprotein particles beneath the endothelium, presumably from increased transport and/or permeability of the lining endothelial cells. This event is rapidly followed by attachment, adherence and spreading of peripheral blood monocytes and T-lymphocytes at sites throughout the arterial tree [1].

The response-to-injury hypothesis of atherosclerosis states that the protective inflammatory response followed by the formation of a fibro proliferative response begins as a protective mechanism that with time and continuing insult may become excessive. According to Nobel prize winner Linus Pauling, the primary cause of human cardiovascular disease is a deficiency in ascorbate leading to the deposition of Lipoproteins -Lp(a)- and fibrinogen/fibrin in the vascular wall [2].

Many genetic defects are associated with ascorbate deficiency. As a result of a genetic defect the rate-constants of certain enzyme-controlled metabolic reactions are decreased. The total depletion of ascorbate in scurvy, leads to a complete loss of the integrity and stability of the vascular wall and to the extravasation of blood into the perivascular area. Ascorbate deficiency leads to an incomplete hydroxylation and thus weakens the extracellular matrix. Alterations of the endothelium and loose connective tissue are known to be characteristic features of atherosclerotic plaques [3].

Another finding central to Pauling's theory was the observation that plaque deposits are not formed randomly throughout the circulatory system. This was first reported in the early 1950s when a Canadian doctor, G. C. Willis, MD, observed that plaque always forms nearest the heart, where blood vessels and arteries are constantly being stretched and bent, rather than being spread evenly throughout the entire cardiovascular system [4]. Willis also noted that plaque deposits always occur in regions that are exposed to the highest blood pressures, such as the aorta, where blood is forcefully ejected from the heart.

This theory states that human atherosclerosis can be primarily a degenerative disease initiated by ascorbate deficiency. This deficiency is the result of the inability of humans to synthesize endogenous ascorbate combined with an insufficient dietary ascorbate intake and/or its destruction by habits like cigarette smoking. It is already known that one cigarette destroys $250 \mathrm{mgs}$ of Vitamin $\mathrm{C}$ in the body.

It is then controversial for some investigators that found useless 
the strategy of lowering LDL for atherosclerosis prevention. The most important of these investigators is the already mentioned Dr Linus Pauling who in 1989 announced a breakthrough in how we view and treat heart disease. In "A Unified Theory of Human Cardiovascular Disease," Linus Pauling announced that the deposits of plaque seen in atherosclerosis were not the cause of heart disease, but were actually the result of our bodies trying to repair the damage caused by long-term vitamin $\mathrm{C}$ deficiency. In essence, Pauling believed that atherosclerosis is a form of scurvy, and plaque is the body's attempt to reinforce and patch weakened blood vessels and arteries that would otherwise rupture. Pauling also showed that heart disease can be prevented or treated by taking vitamin $\mathrm{C}$ and other micronutrients.

Putting all the pieces of the puzzle together, Pauling suggested that the ability to form plaque is really the body's attempt to repair damage caused by a long-term deficiency of vitamin C.

\section{The Role of Heavy Metals}

All metals are toxic at higher concentrations. Excessive levels can be damaging to the organism. Other heavy metals such as mercury, plutonium, and lead are toxic metals that have no known vital or beneficial effect on organisms, and their accumulation over time in the bodies of animals can cause serious illness.

Certain elements that are normally toxic are for certain organisms or under certain conditions, beneficial. Examples include vanadium, tungsten, and even cadmium.

There are more than 30 metals that are of concern for environmental authorities because of residential or occupational exposure, out of which 23 are heavy metals: antimony, arsenic, bismuth, cadmium, cerium, chromium, cobalt, copper, gallium, gold, iron, lead, manganese, mercury, nickel, platinum, silver, tellurium, thallium, tin, uranium, vanadium, and zinc. These heavy metals are commonly found in the environment and diet. In small amounts they are required for maintaining good health but in larger amounts they can become toxic or dangerous. Heavy metal toxicity can lower energy levels and damage the functioning of the brain, lungs, heart, blood vessels, kidney, liver, blood composition and other important organs. Repeated long-term exposure of some metals and their compounds may even cause cancer [5].

Population studies have demonstrated a link between lead exposure and subsequent development of hypertension and cardiovascular disease. In vivo and in vitro studies have shown that chronic lead exposure causes HTN and cardiovascular disease by promoting oxidative stress, limiting nitric oxide availability, impairing nitric oxide signalling, augmenting adrenergic activity, increasing endothelin production, altering the renin-angiotensin system, raising vasoconstrictor prostaglandins, lowering vasodilator prostaglandins, promoting inflammation, disturbing vascular smooth muscle $\mathrm{Ca}(2+)$ signalling, diminishing endotheliumdependent vasorelaxation, and modifying the vascular response to vasoactive agonists [5]. Moreover, lead has been shown to cause endothelial injury, impede endothelial repair, inhibit angiogenesis, reduce endothelial cell growth, suppress proteoglycan production, stimulate vascular smooth muscle cell proliferation and phenotypic transformation, reduce tissue plasminogen activator, and raise plasminogen activator inhibitor-1 production
[6-9]. Via these and other actions, lead exposure causes hypertension and promotes atherosclerosis, thrombosis, and cardiovascular disease.

Cadmium is a by-product of zinc production which humans or animals may get exposed to at work or in the environment. Once this metal gets absorbed by humans, it will accumulate inside the body throughout life. This metal was first used in World War I as a substitute for tin and in paint industries as a pigment. In today's scenario, it is also being used in rechargeable batteries, for special alloys production and also present in tobacco smoke. Cadmium is a highly toxic nonessential heavy metal that is well recognized for its adverse influence on the enzymatic systems of cells, oxidative stress and for inducing nutritional deficiency [5].

Smokers are more susceptible for cadmium intoxication than non-smokers. Tobacco is the main source of cadmium uptake in smokers as tobacco plants, like other plants, can accumulate cadmium from the soil. Non-smokers are exposed to cadmium via food and some other pathways [10]. Studies in the last decade have shown that cadmium levels in blood and urine are independent factors associated with the development of atherosclerotic plaques at baseline as well as prospectively. This novel observation emphasizes the need to consider cadmium as a proatherogenic pollutant [11].

Heavy metal contamination of vegetables cannot be underestimated as these foodstuffs are important components of human diet. Vegetables are rich sources of vitamins, minerals, and fibres, and also have beneficial antioxidative effects. However, intake of heavy meta-contaminated vegetables may pose a risk to the human health. Heavy metal contamination of food is one of the most important aspects of food quality assurance. Heavy metals are nobiodegradable and persistent environmental contaminants, which may be deposited on the surfaces and then absorbed into the tissues of vegetables. Monitoring and assessment of heavy metals concentrations in the vegetables from the market sites have been carried out in some developed and developing countries.

\section{Vitamin K2, the "new kid on the block"}

Research published in Expert Review of Clinical Pharmacology revealed that, in contrast to the current belief that cholesterol reduction with statins decreases atherosclerosis, the drugs may instead actually stimulate atherosclerosis and heart failure [12]. There were several physiological mechanisms discussed in the study that show how statin drugs may make your heart health worse; one being that they inhibit the synthesis of vitamin K2. Vitamin K2 protects your arteries from calcification. Without it, plaque levels worsen.

Vitamin K2's biological role is to help move calcium into the proper areas in your body, such as your bones and teeth. It also plays a role in removing calcium from areas where it shouldn't be, such as in your arteries and soft tissues [13].

Vitamin K2 is associated with reduced vascular calcification even at small dietary intakes. Statin drugs inhibit the function of vitamin $\mathrm{K} 2$ in your body, which means taking them may put you at risk of vitamin K2 deficiency, a condition known to contribute to a number of chronic diseases, including: Osteoporosis, Heart disease, Heart attack and stroke, Inappropriate calcification 
from heel spurs to kidney stones, Brain disease, Cancer.

More than seven decades ago, while investigating the effects of a low-fat diet fed to chickens, Danish scientist Henrik Dam discovered vitamin $\mathrm{K}$. He found that the bleeding tendencies found in the chickens on that diet could be prevented when a diet with normal levels of fat was restored and vitamin $\mathrm{K}$ was added to their diets. From that point forward, vitamin $\mathrm{K}$ became known as the coagulation vitamin, the " $K$ " coming from the German word koagulation.

Vitamin $\mathrm{K}$ is not a single entity but, rather, a family of structurally related molecules derived from different sources. There are two main forms of the vitamin. Phylloquinone (K1) is found in leafy green plants and menaquinone (K2) is found in animal meat and fermented foods [13].

Since 1950, the consumption of vitamin K has decreased gradually, and even a well-balanced diet might not provide vitamin $\mathrm{K}$ in amounts sufficient for satisfying the body's needs. The population based Rotterdam study studied 4807 healthy men and women older than age 55 years, evaluating the relationship between dietary intake of vitamin $\mathrm{K}$ and aortic calcification, heart disease, and all-cause mortality. The study revealed that high dietary intake of vitamin K2-at least $32 \mathrm{mcg}$ per day, with no intake of vitamin $\mathrm{K} 1$, was associated with a $50 \%$ reduction in death from cardiovascular issues related to arterial calcification and a $25 \%$ reduction in all-cause mortality [14].

The entire skeleton is replaced every 7 to 10 years. During the skeleton's remodeling, the body releases calcium from the bone into the bloodstream to meet an individual's metabolic needs, allowing the bone to alter size and shape as it grows or repairs from injuries. This remodeling is regulated by osteoblasts-cells that build up the skeleton-and osteoclasts-cells that break down the skeleton. Osteoblasts produce osteocalcin, which helps take calcium from the blood circulation and bind it to the bone matrix. The newly made osteocalcin, however, is inactive, and it needs vitamin $\mathrm{K} 2$ to become fully activated and bind calcium.

That requirement alone makes vitamin $\mathrm{K} 2$ a major player in bone health, but its importance does not stop there. Vitamin K2 also keeps calcium from accumulating in the walls of blood vessels. The vitamin K-dependent protein, matrix GLA protein, is a central calcification inhibitor produced by the cells of vascular smooth muscles and regulates the potentially fatal accumulation of calcium [15].

\section{Periodontal Disease}

Several infectious diseases have been implicated as possibly causing inflammation and heart disease. Three mutually exclusive periodontal classifications were defined based on the Russell Periodontal Index: periodontitis, gingivitis, and periodontal health [16]. Several biological mechanisms through which periodontal disease may cause CHD have been proposed as follows: the invasion into endothelial coronary cells by oral microorganisms the harmful cardiovascular effects of inflammatory response to periodontitis, or contributions of oral microorganisms to acute thromboembolic events during bacteremia [16-18].

Infectious agents might conceivably furnish inflammatory stimuli that accentuate atherogenesis. Acute infections can alter hemodynamics and the clotting and fibrinolytic systems in ways that can precipitate ischemic events. Chronic extravascular infections (eg, gingivitis, prostatitis, bronchitis) can augment extravascular production of inflammatory cytokines that may accelerate the evolution of remote atherosclerotic lesions. Many human plaques show signs of infection by microbial agents such Chlamydia pneumoniae. Chlamydiae, when present in the arterial plaque, may release lipopolysaccharide (endotoxin) and heat shock proteins that can stimulate the production of proinflammatory mediators by vascular endothelial cells and SMCs and infiltrating leukocytes alike [19].

Dental disease is associated with an increased risk of coronary heart disease, particularly in young men. Whether this is a casual association is unclear. Common oral periodontal bacteria, that is, Prevotella gingivalis and Prevotella intermedia, may contribute to the injury and inflammation that provokes atherosclerosis. Chronic oral infections such as adult periodontitis, may have importante long-term sequelae. Aactinobacillus actinomycetemcomitans, Porphyromonas gingivalis, Bacteroides forsythus and Prevotella intermedia have been detected in atheromatous plaques by PCR. It has also been recognized thatperiodontal diseases, which are chronic gram-negative infections, represent a risk factor for atherosclerosis and thromboembolic events. Periodontal disease, once established, provides a biological burden of endotoxin and inflammatory cytokines which serve to initiate and exacerbate atherogenesis and thromboembolic events. Periodontitis and atherosclerosis have complex etiologies, genetic and gender predispositions, and potentially share many risk factors, the most significant of which may be smoking status $[20,21]$.

\section{Conclusions}

Atherosclerosis is not a single disease entity. In fact, the lesions of atherosclerosis represent a common response of the artery to numerous and potentially different forms of insult. Examination of atherosclerotic lesions reveals that each lesion contains the elements of an inflammatory response together with varying levels of fibro proliferative response.

Numerous prospective epidemiological studies have identified a direct relationship between serum total cholesterol level and coronary heart disease incidence. Since the Framingham study it has long been considered common knowledge that total serum cholesterol is a strong independent risk factor for cardiovascular disease. During the last decades, cardiovascular disease prevention has been marked by a trend of gradually lowering thresholds of cholesterol levels and campaigns aimed at the general public have underlined the risks associated with total cholesterol above 5.0 mmol L-1.

Current practice includes clinical assessment and the results from routine chemical assays deriving LDL-cholesterol concentration by the Friedewald calculation [LDL-cholesterol = total cholesterol - HDL-cholesterol - (TG/2.2) in mmol/L]. In the vast majority of prevention centers, the patients undertake blood samples to measure total cholesterol, HDL and triglycerides, and the LDL, the real culprit of the problem in the cholesterol theory, is calculated using a formula. It is the oxidized LDL which enters the arterial wall, and not free cholesterol. As we know, choles- 
terol is not water soluble enough and it is not entitled to travel free within the bloodstream. It looks quit simplistic and up to date quite useless, basing treatments for dyslipidemia on a statement:"the lower, the better".

The HUNT 2 study21, a very elegant prospective study in Scandinavia included 52.087 Norwegians, aged 20-74, that were followed-up on cause-specific mortality for 10 years. This is 510.297 person-years in total. The statistical power and the lack of conflict of interest make this a heavy evidence source, even stronger than Framingham. Based on their results, they clearly contradict the popularized idea of a positive, linear relationship between cholesterol and fatal disease, and conclude that the underlying assumptions regarding cholesterol in clinical guidelines for prevention might be flawed. This study could suggest that, like any other substance in the body, like thyroid hormone for instance, total cholesterol should be in a balanced situation, not too high, not too low. According to their presented results, between 5 and $7 \mathrm{mmol} \mathrm{L-1}$ would be the desired level. They reported an interesting low mortality rate among post-menopausal women with a mean cholesterol level of $7 \mathrm{mmol} \mathrm{L-1.}$

Diabetes, smoking, hypertension, family history are well established coronary risk factors. But we must open the box and look outside so we can stop missing additional culprits of cardiovascular disease in current times.

\section{References}

1. Moore KJ, Tabas I. The Cellular Biology of Macrophages in Atherosclerosis. Cell. 2011;145(3):341-355. doi: 10.1016/j.cell.2011.04.005

2. Rath M, Pauling L. Solution to the Puzzle of Human Cardiovascular Disease: Its Primary Cause is Ascorbate defiency, leading to the deposition of lipoprotein(a) and fibrinogen / fibrin in the vascular wall. J Orthomol Med. 1991;6:125-134.

3. Rath M, Pauling L. Immunological evidence for the accumulation of lipoprotein(a) in the atherosclerotic lesion of the hypoascorbemic guinea pig. Proc Natl Acad Sci U S A. 1990;87(23):9388-9390.

4. Willis GC, Light AW, Gow WQS. Serial Arteriography in Atherosclerosis. Can Med Assoc J. 1954;71(6):562-568.

5. Jaishankar M, Tseten $\mathrm{T}$, Anbalagan $\mathrm{N}$, Mathew $\mathrm{BB}$, Beeregowda KN. Toxicity, mechanism and health effects of some heavy metals. Interdiscip Toxicol. 2014;7(2):60-72. doi: 10.2478/intox-2014-0009.

6. Vaziri ND. Mechanisms of lead-induced hypertension and cardiovascular disease. Am J Physiol Heart Circ Physiol. 2008;295(2):H454-65. doi: 10.1152/ajpheart.00158.2008

7. Kishimoto T, Oguri T, Ueda D, Tada M. Effect of lead on tube formation by cultured human vascular endothelial cells. Arch Toxicol. 1995;69(10):718-721.
8. Kaji T, Ohkawara S, Nakajima M, Yamamoto C, Fujiwara Y, Miyajima S, et al. Lead-induced alteration of heparan sulfate proteoglycans in cultured vascular endothelial cells. Toxicology. 1997;118(1):1-10.

9. Kaji T, Suzuki M, Yamamoto C, Mishima A, Sakamoto M, Kozuka H. Severe damage of cultured vascular endothelial cell monolayer after simultaneous exposure to cadmium and lead. Arch Environ Contam Toxicol. 1995;28(2):168-172.

10. Emman A, Gordon F. Heavy metal poisoning and Cardiovascular Disease. Journal of toxicology. 2011.

11. Fagerberg B, Bergstrom G, Boren J, Barregard L. Cadmium exposure is accompanied by increased prevalence and future growth of atherosclerotic plaques in 64-year-old women. J Intern Med. 2012;272(6):601-610. doi: 10.1111/j.13652796.2012.02578.x

12. Okuyama H, Langsojen $\mathrm{P}$, Hamazaki T, Ogushi $\mathrm{Y}$, Hama $R$, Kobayashi T, Uchino $H$. Statins stimulate atherosclerosis and heart failure: pharmacological mechanisms. Expert Rev Clin Pharmacol. 2015;8(2):189-199. doi: 10.1586/17512433.2015.1011125

13. Beulens JW, Bots ML, Atsma F, Bartelink ML, Prokop M, Geleijnse JM, et al. High dietary menaquinone intake is associated with reduced coronary calcification. Atherosclerosis. 2009;203(2):489-493. doi: 10.1016/j.atherosclerosis.2008.07.010

14. Geleijnse JM, Vermeer C, Grobbee DE, Schurgers LJ, Knapen $\mathrm{MH}$, van der Meer IM, et al. Dietary intake of menaquinone is associated with a reduced risk of coronary heart disease: the Rotterdam Study. J Nutr. 2004;134(11):3100-3105.

15. Zheng S, Zhangsuo L, Donghai W. The efficacy of vitamin $\mathrm{K}$ on vascular calcification for chronic renal failure patient receiving dialysis. Int J Clin Exp Med. 2016;9(9):18092-18097.

16. Russell AL. A system of scoring for prevalence surveys of periodontal disease. J Dent Res. 1956;35(3):350-359. DOI: $10.1177 / 00220345560350030401$

17. Dorn BR, Dunn Jr WA, Progulske-Fox A. Invasion of human coronary artery cells by periodontal pathogens. Infect Immun. 1999;67(11):5792-5798.

18. Deshpande RG, Khan MB, Genco CA. Invasion of aortic and heart endothelial cells by Porphyromonas gingivalis. Infect Immun. 1998;66(11):5337-5343.

19. Beck JD, Offenbacher S. Systemic effects of periodontitis: epidemiology of periodontal disease and cardiovascular disease. J Periodontol. 2005;76(11 Suppl):2089-2100. 
20. Mustapha IZ, Debrey S, Oladubu M, Ugarte R. Markers of systemic bacterial exposure in periodontal disease and cardiovascular disease risk: a systematic review and meta-analysis. J Periodontol. 2007 Dec;78(12):2289-2302.
21. Petursson H, Sigurdsson JA, Bengsson C, Nilsen T, Gletz L Is the use of cholesterol in mortality risk algorithms in clinical guidelines valid? Ten years prospective data from the Norwegian HUNT 2 study. J Eval Clin Pract. 2012;18(1):159-168. doi: 10.1111/j.1365-2753.2011.01767.x 